\title{
RETRACTED ARTICLE: Deep network for visual saliency prediction by encoding image composition
}

\author{
Bo Dai ${ }^{1} \cdot$ Weijing $\mathrm{Ye}^{1} \cdot \operatorname{Jing} \mathrm{Zheng}^{1} \cdot$ Caiyou Zhang ${ }^{1}$
}

Received: 12 October 2017 /Revised: 30 October 2017 / Accepted: 5 November 2017 /

Published online: 12 November 2017

(C) Springer Science+Business Media, LLC, part of Springer Nature 2017

The Editor-in-Chief has retracted this article [1], which was published as part of special issue "Multi-source Weak Data Management using Big Data", because it shows substantial text overlap, most notably with the articles cited $[2,3]$. In addition, there is evidence suggesting authorship manipulation and an attempt to subvert the peer review process.

The authors have not responded to correspondence about this retraction.

\section{References}

1. Dai, B., Ye, W., Zheng, J. et al. Deep network for visual saliency prediction by encoding image composition. Multimed Tools Appl (2017). https://doi.org/10.1007/s11042-017$5400-8$

2. Zhang, L., Xia, Y., Ji, R. et al. Spatial-aware object-level saliency prediction by learning graphlet hierarchies. IEEE Transactions on Industrial Electronics (2014). https://doi. org/10.1109/TIE.2014.2336602

3. Zhang, L., Li, X., Nie, L. et al. Weakly supervised human fixations prediction. IEEE Transactions on Cybernetics (2015). https://doi.org/10.1109/TCYB.2015.2400821

Electronic supplementary material The online version of this article (https://doi.org/10.1007/s11042-0175400-8) contains supplementary material, which is available to authorized users.

Caiyou Zhang

zhangcaiyou1778@126.com

1 State Grid Zhejiang Electric Power Company, Information\&telecommunication Branch, Hangzhou, China 